NOTICE

PORTIONS OF THIS REPORT ARE IULGIBLE. It has been reproduced from the bessible avait ability.

\title{
Radial Particle Distributions in PARMILA Simulation Beams
}

\author{
G. P. Boicourt
}


RADIAL PARTICLE DISTRIBUTIONS IN PARMILA SIMULATION BEAMS

by

G. P. Boicourt

\section{ABSTRACT}

The estimation of beam spill in particle accelerators is becoming of greater importance as higher current designs are being funded. To the present, no numerical method for predicting beam-spill has been available. In this paper, we present an approach to the loss-estimation problem that uses probability distributions fitted to particle-simulation beams. The properties of the PARMILA code's radial particle distribution are discussed, and a broad class of probability distributions are examined to check their ability to fit it. The possibility that the PARMILA distribution is a mixture is discussed, and a fitting distribution consisting of a mixture of two generalized gamma distributions is found. An efficient algorithm to accomplish the fit is presented. Examples of the relative prediction of beam spill are given.

\section{INTRODUCTION}

Particle simulation codes are widely used to design accelerators and to predict the performance of accelerators under various operating conditions. One of the operating values of an accelerator is the amount of beam lost during the transit of the accelerator. At the present time, there appears to be no reasonable numerical method for predicting this value, and the accelerator designer is faced with a nasty problem. He is asked to design an accelerator that has spill below a specified level but has no means to check his design except by testing the finished product. Under the circumstances, he would be quite happy if he had a means to predict, a priori, even the order of magnitude of the beam spill. One numerical approach to this problem is to find an analytical expression for the radial particle distribution of the simulation 
beam. We need the radial distribution because the bores of most linear acce1erators are circular, and we want to predict the amount of the beam at, or outside, the circular aperture because the losses should be proportional to the amount calculated outside. Some corrections are required for off-center beams. The amount outside is only proportional because, first, there are breaks in the bore--for instance, gaps between drift tubes; and second, there must be some characteristic diffusion time to replenish the tail once it has been wiped off by striking the bore.

Once the analytical distribution is available, it provides directly a means for evaluating the relative effect on spill of mechanical misalignments, quadrupole harmonics, and oti:er variables of operation. All that is required is for us to run the simulation code with and without the change and to compare the relative amount of current predicted at or beyond the bore radius.

Some have objected that, although the simulation codes are recognized as good representations of the core of the beam, there is no guarantee that the simulated tails of the beam are anything like reality. The objection is only partially valid. It is true that no code is better than the physical approximations made in the computation; in this sense, if some of the physics is left out, the tail will be incorrectly computed, as will the core. However, the core as computed by PARMILA* generally is accepted to be a fairly good description of reality, and the same physics the code used to produce the core of the beam also is used to produce the tail. In this sense, simulation of the tail is just as good as that of the core. To use the collective information contained in the simulation beam properly, we must fit the entire distribution, not just the tail, because the distribution of particles in the core has a bearing on the distribution of particles in the tail.

Simulation codes like PARMILA rely on a small number of pseudoparticies to represent the beam and, therefore, do not provide sufficient resolution to

\footnotetext{
*PARMILA (Phase And Radial Motion In Linear fccelerators) is a versatile, modularized multiparticle simulation code in which each farticle is transported through the accelerator elements by the chain-matrix method. The treatment is fully 6D, and the code contains highly detailod, nonlinear modeling. The space-charge model is an $r-z 20$ particle-in-cell method that gives careful attention to details such as charge distribution and refitting of the mesh along the machine. A version of the code has been configured to run very large particle distributions ( $>50$ 000 particles) on the CRAY computer to address the present study with better accuracy.
} 
predict losses at the current levels required. Thus, in the FMIT (Fusion Materials Irradiation Test) linac, the design goal is less than $3 \mu \mathrm{A} / \mathrm{m}$. For a computer run accelerating $100 \mathrm{~mA}$ and using 50000 particles to represent the beam, each oarticle corresponds to $2 u A$. Because there are about two cells per meter in the middle of the linac, a loss of even one pseudoparticle in a cell corresoonds to a Der-meter loss of 4 iA--already over the goal. We use a fitted distribution to solve this numerical resolution problem.

It is orobably wise to comment on one commonly used particle distribution before orocesding. The normal distribution often is used to describe the beam in some sense. For instance, we know that the number of particles found at several standard deviations (a normal distribution parameter) is much larger than we would find there if the distributions were normal. This observation has been used to throw doubt on PARMILA calculations because the claim has been that the core of the PARMILA beam is close to normal. Close checks of the PARMILA beam when a substantial number of simulation particles are run show that PARMILA distributions also have too many particles in the tail to be described by the normal distribution, even though at first glance the beam core is fairly close to norna?. We will see later that the real problem is not that the tail is toc long for the distribution but, rather, that the peak is too high. The attempt to fit a normal distribution to a peak too high for it results in a drawing-in of the tail. The normal distribution simply is not correct for describing the distribution in either of the transverse coordinates.

In this report, I shall describe what has been done to find an easily calculable probability distribution to fit the PARMILA radial ieam distribution. There are a lot of loose ends, and I hope that some of the details below will help others should they pursue this further, either by letting them know what has been done or by pointing to some things that need to be dorie. The , fitting distribution finally obtained is adequate in that it seems to predict the tail to within about a faccor of 2, which is considerably better thair an order of magnitude. More specifically, the distritution seems able to predict a tail comprising only $0.2 \%$ of the total beam to within a factor of 2 . The

\footnotetext{
* The FMIT deuteron DTL (drift-tube linac) accelerates 100-MA average beam current from 2 to $35 \mathrm{Mel}$ and transports the output beam to a molten lithium target.
} 
distribution also is fäirly easy to compute though not straightforward. It can be, and has been, incorporated into a version of the PARMILA code so that relative effects of system changes on spill can be obtained as a by-product of a run. It is not the final word, if for no other reason than because a completely consistent physical justification for its use has not been given.

In Sec. II the statistical properties of the PARMILA radial beam distribution are examined, and some of the implications are discussed. Section III lists and discusses the group of pure probability distributions chat were tried as candidates for the fitting distribution. Section IV describes the mixed distribution that (almost) fits the PARMILA radial distribution. The next section, $V$, presents an algorithm that finds the mixed distribution parameters from the PARMILA distribution. In Sec. VI some examples are given to illustrate the use of the fitted distribution to get relative measures of the effects of system changes on spill, and $i$ in Sec. VII I discuss some possibilities as to how the fitted distribution might be used to predict beam spill quantitatively.

II. PROPERTIES OF THE PARMILA RADIAL BEAM DISTRIBUTION

In the search for an analytical distribution, knowledge of the properties of the PARMILA beam distribution can help to fit it by narrowing the possibilities. We can say quite a bit about what will be required from the fitting distribution. First, it must be zero at the origin (the centroid of the beam), not because there cannot be particles there, but because the probability in a radial distribution is proportional to the area and the area goes to zero. Next, the distribution must be a parent distribution to the Weibull distribution. That is, let the cumulative distribution function of the fitting distribution be $F(r)$ and let $W(r)$ be a Weibull distribution; then, $F(r)$ is a parent Jistribution to $W(r)$ if $1-[1-F(r)]^{n}=W(r)$ when $n$ is 1 arge. ${ }^{1,2}$ Boicourt and Jameson ${ }^{3}$ have shown that the distribution of largest values for the PARMILA radial distribution is Weibull, and this condition is equivalent to the requirement that the fitting distribution be a parent to the weibull.

The distribution chosen to fit the radial particle distribution should have the capability of fitting the range of distribution moments displayed by the particle distribution. Two important measures of any distribution are its skewness $B_{1}$ and its kurtosis or peakedness $B_{2}$. There are several systems of distributions designed to cover ranges in the skewness-kurtosis plane, the most 
well known of which is the Pearson system." The Pearson chart of the $B_{1}-B_{2}$ plane gives us a convenient way to examine some of the required moment properties of the fitting distribution. Figure 1 shows a chart of the $\beta_{1}-\beta_{2}$ plane with the points, lines, and areas covered by some distributions. The normal distribution is at the point $(0 ., 3$.$) . The normal distribution usually is$

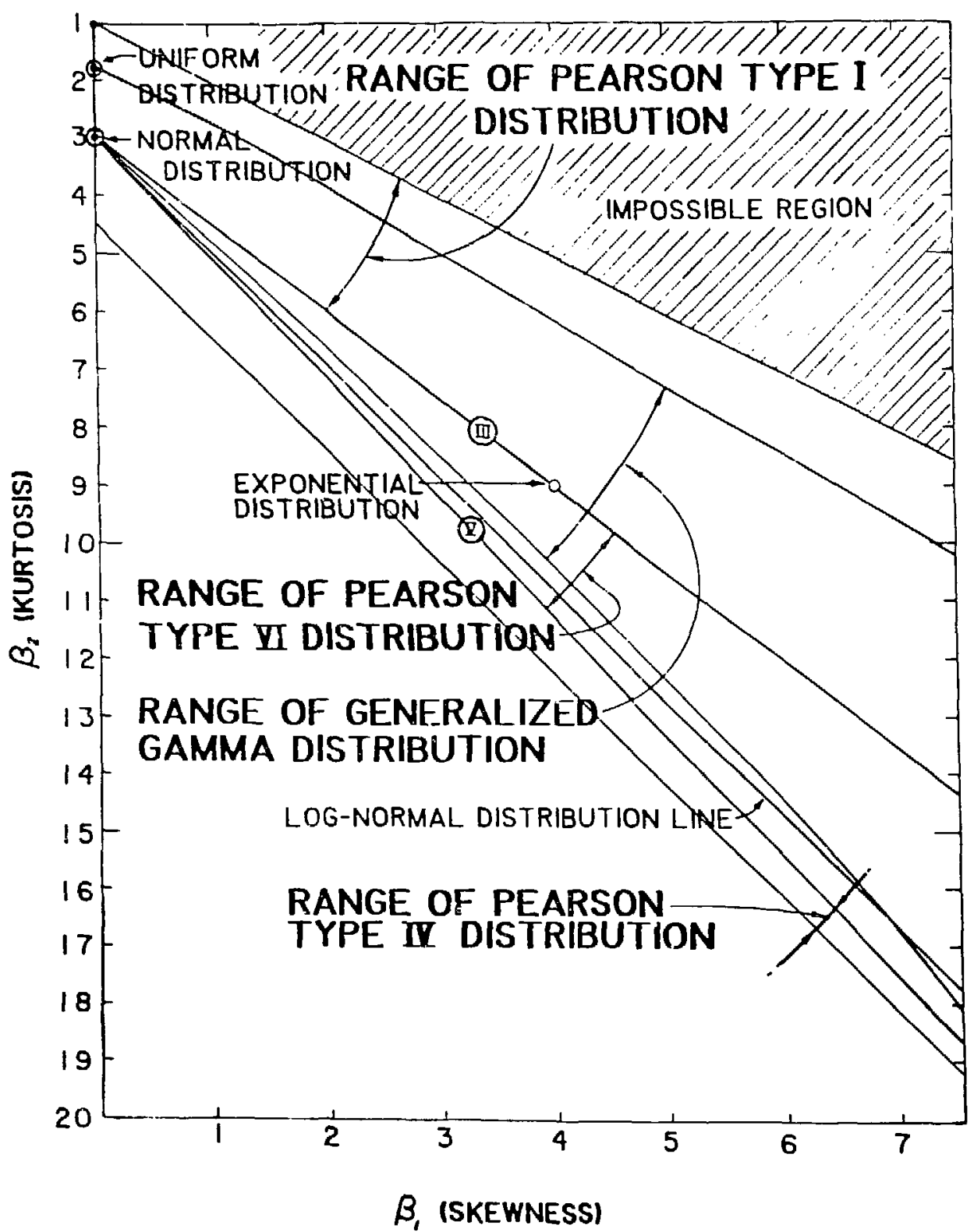

Fig. 1. $B_{1}-B_{2}$ plane showing regions corresponding to certain distributions. 
considered to be medium peaked, that is, mesokurtic. ${ }^{5}$ More peaked distributions are called leptokurtic and less peaked distributions are called platykurtic. The $\left(\beta_{1}, \beta_{2}\right)$ points found for the PARMILA radial distribution of a $100-m A$ beam at 76 positions in the FMIT deutron linac are plotted on the $\beta_{1}-\beta_{2}$ plane in Fig. 2. Almost all of the radial beam distributions are leptokurtic and this fact justifies the statements about normal-distribution peak height made in the previous section. Except for two of the points shown in Fig. 2, the distributions were taken at the end of cells in the linac. It is of interest to plot the $\beta_{1}$ and $\beta_{2}$ values versus cell number in the linac. The plots shown in Fig. 3 are for a zero-current beam (that is, no space-charge forces were calculated), and they plainly show a periodicity as the beam proceeds down the linac. The input beam was generated by a simulation run through an RFQ (radiofrequency quadrupole) and was transversely matched to the DTL in an average sense. ${ }^{6}$ The transverse profiles after matching are quite smooth. Figure 4 is a similar plot for a 100-mA beam. Here, some of the periodicity has been

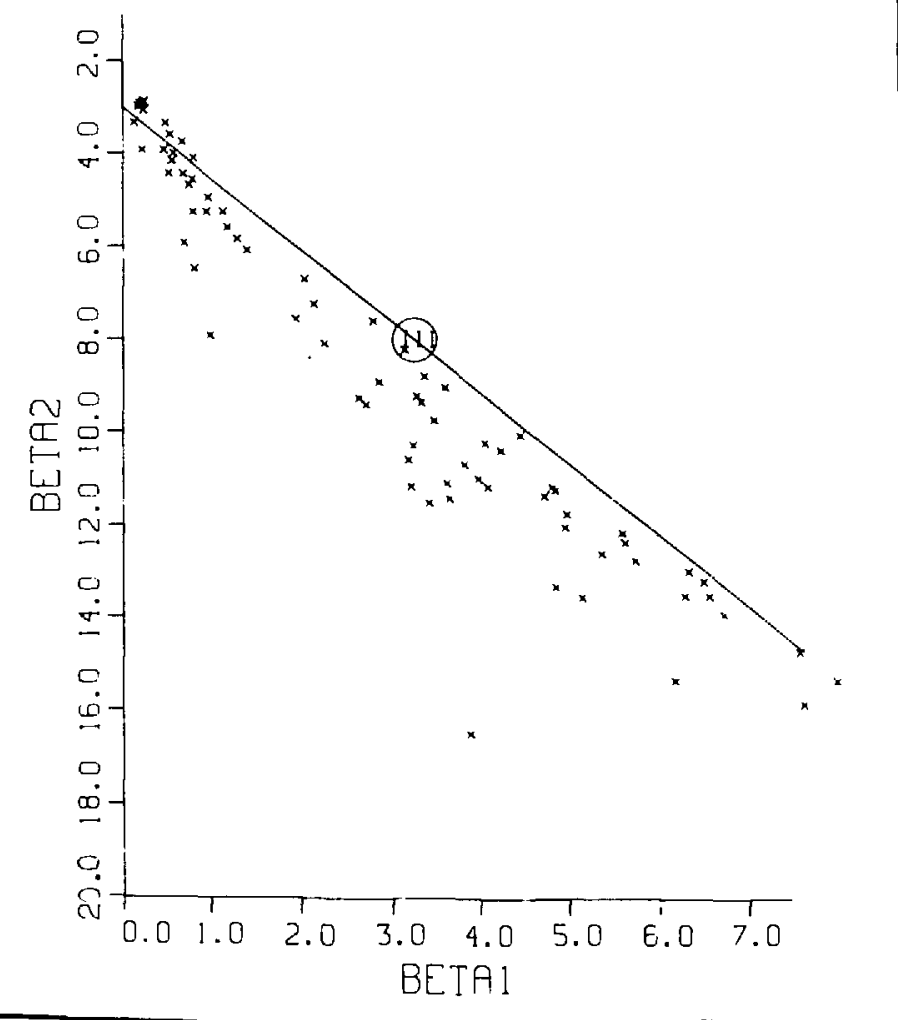

Fig. 2. Location in the $R_{1}-B_{2}$ plane of $\left(B_{1}, B_{2}\right)$ points obtained in one run through the FMIT DTL section. 

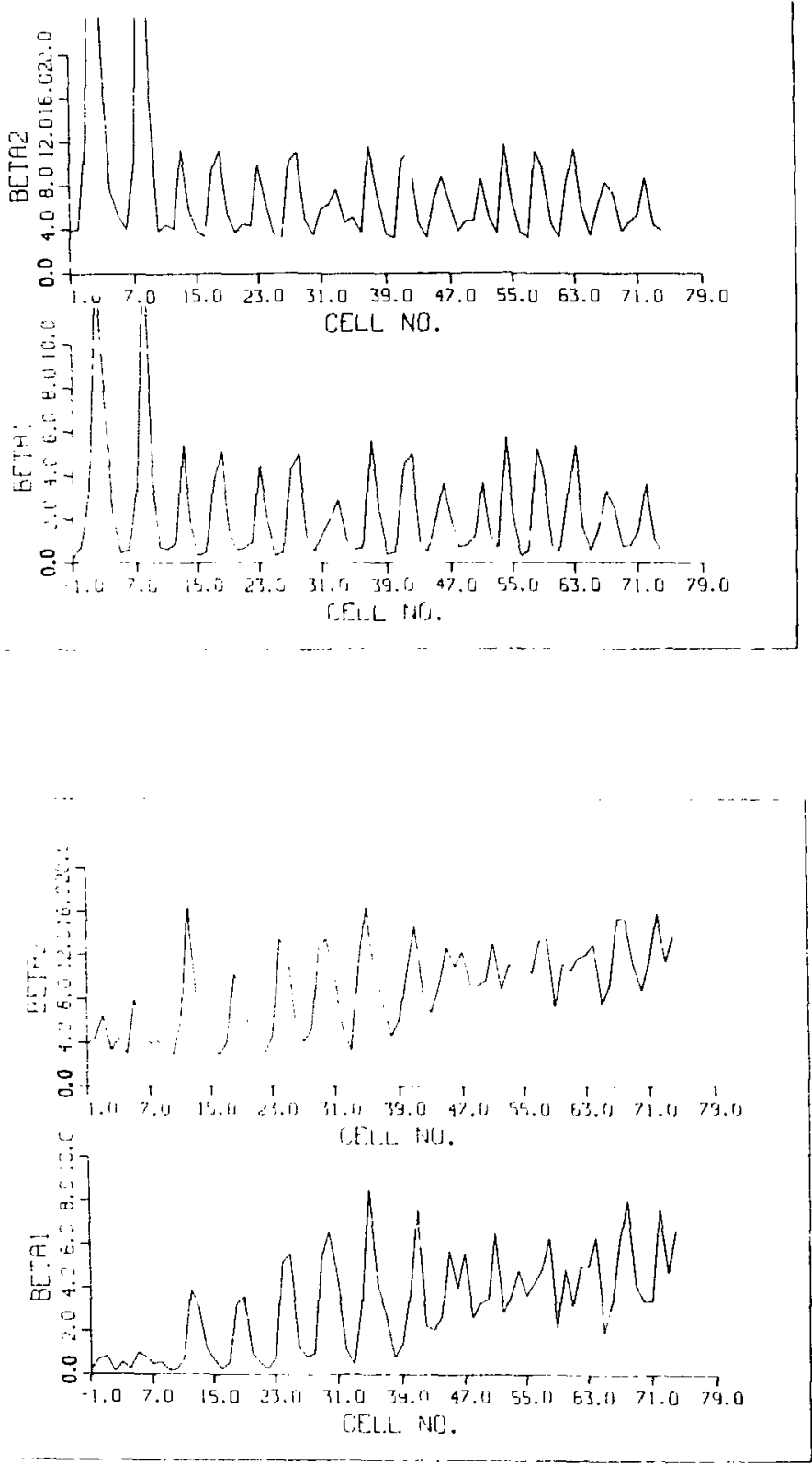

Fig. 3. Plot of skewness $B$ ] and kurtos is $B_{2}$ versus cell number obtained in one zerocurrent run through the FMIT DTL section.
Fig. 4. Plot of skewness $\beta$ and kurtosis $B_{2}$ versus cell number for a $100-\mathrm{mA}$ run through the FMIT DTL section.

lost, but now we also see a growth in both $\beta_{1}$ and $\beta_{2}$ toward the downstream end of the linac. In the 100-mA case, the values start smaller than for zero current but they grow. I have no good physical explanation for these observations, but their presence indicates a connection between the physics of the machine and the radial distribution. 
The information above indicates that, at least for a pure fitting distribution as opposed to a mixture, we want a distribution that is zero at the origin, that probably goes to zero exponentially at infinity, and that is capable of being strongly leptokurtic. In the next section I describe some of the distributions tried.

\section{DISTR IBUTIONS EXAMINED}

The above requirements seem to tell us all we need to know to find the proper distribution, provided it is a pure distribution and not a mixture distribution. We hope to find a fairly simple, well-known probability distribution that fits the PARMILA radial distribution. "Simple and well known" implies the fitting methods are usually simple and fast. In the search, we should not arbitrarily rule out a distribution merely because it does not cover the precise reqion in the skewness-kurtosis plane. This is so for two reasons. First, because our ultimate aim is to bound the radial distribution tail for loss predictions, an exact fit is not necessarily required so long as the predicted tail consistently is sufficiently close to the observed tail. Second, it is common practice to use simpler and more tractable distributions as approximations for our type of prediction. To quote Johnson and Kotz: "The principal aim in choosing one of these forms of distribution is to facilitate the mathematical analysis to which it is to be subjected, while attaining a reasonable approximation." 4

Another complication arises because information is not necessarily available on what portion of the skewness-kurtosis plane is covered by a given distribution. In these cases, often it is easier just to try the distribution instead of finding the region first and then, in light of the previous comments, trying the distribution even if it covers the wrong region but is somewhere close.

It is not even possible to throw out double-tailed distributions because it may turn out that the probabilities in the tail at and below the origin are so small they can be ignored.

Table I is a list of individual distributions, either actually tried or examined and rejected without trial for some reason. There is some overlap in the group because usually a simpler form was tried first for the reasons given above. 
Below, we comment on the ability of the distributions in Table I to fit the PARMILA, radial distribution.

TABLE I

\section{LIST OF DISTRIBUTIONS EXAMINED}

Name

Probability Density Function

Bivariate Normal

$\frac{r}{2 \pi \sigma_{x} \sigma_{y} \sqrt{\left(1-\rho^{2}\right)}} \int_{0}^{2 \pi} \exp [-Q(r, \theta)] d \theta$

$Q(r, \theta)=\frac{r^{2}}{2\left(1-\rho^{2}\right)}\left(\frac{\cos ^{2} \theta}{\sigma_{x}^{2}}-\frac{2 p}{\sigma_{x} \sigma_{y}} \sin \theta \cos \theta+\frac{\sin ^{2} \theta}{\sigma_{y}^{2}}\right)$

. $\sigma_{x}, \sigma_{y}>0,-1<\rho<1,0 \leq \theta<2 \pi, 0<r<\infty$

Birnbaum-Saunders

$$
\frac{1}{2 \sqrt{2 \pi}} \cdot \frac{1}{\alpha r}\left[\left(\frac{r}{\beta}\right)^{1 / 2}+\left(\frac{\beta}{r}\right)^{1 / 2}\right] \exp \left[-(r / \beta+\beta / r-2) / 2 \alpha^{2}\right]
$$

$\alpha, \beta,>0 \quad 0<r<\infty$

Chi

(Rayleigh, Maxwell)

$2\left(\frac{\alpha}{\beta}\right)^{\alpha} \cdot \frac{1}{\Gamma(\alpha)} r^{2 \alpha-1} \exp \left[-(\alpha / \beta) r^{2}\right]$

$\alpha, \beta>0,0 \leq r<a$

Gamma

(Chi Square)

$\frac{1}{\theta^{\alpha}} \cdot \frac{r^{\alpha-1}}{\Gamma(\alpha)} \exp (-r / \theta)$

$\forall, \alpha>0, r \geq 0$

Generalized Gamma

$$
\begin{aligned}
& \gamma\left(\frac{\alpha}{\beta}\right)^{\alpha} \frac{r^{\gamma \alpha-1}}{I^{\prime}(\alpha)} \exp \left[-(\alpha / \beta) r^{\gamma}\right] \\
& \alpha, \beta, \gamma>0 ; r \geq 0
\end{aligned}
$$


Generalized Lambda

$$
\begin{aligned}
& f(p)=\lambda_{2}\left[\lambda_{3} p^{\lambda_{3}-1}+\lambda_{4}(1-p)^{\lambda_{4}-1}\right]^{-1} \\
& r=\lambda_{1}+\left[p^{\lambda_{3}}-(1-p)^{\lambda_{4}}\right] / \lambda_{2} \\
& -\infty<\lambda_{1}, \lambda_{2}, \lambda_{3}, \lambda_{4}<\infty
\end{aligned}
$$

Gumbel I Smallest Extreme

$$
\begin{aligned}
& \frac{1}{\alpha} \exp [-(r-\gamma) / \alpha] \cdot \exp \{-\exp [(r-\gamma) / \alpha]\} \\
& \alpha>0 ;-\infty<r<+\infty
\end{aligned}
$$

Gumbel I Largest Extreme

$\frac{1}{\alpha} \exp [-(r-\gamma) / \alpha] \exp \{-\exp [-(r-\gamma) / \alpha]\}$

$$
\alpha>0 ;-\infty<r<+\infty
$$

Gumbel II Largest Extreme

$$
B a^{B} r^{-(\beta+1)} \exp [-(\alpha / r) \beta]
$$

$$
r \geq 0 ; \alpha, \beta>0
$$

LaGrange Gamma

$\sum_{k=0}^{j-1} \frac{a[(1+k \theta) a r]^{k-1}[(1+k \theta) a r-k] \exp [-(1+k \theta) a r]}{k !}$

$$
a>0, j=1,2, \ldots ; \theta>1 / j, r \geq 0
$$

Log Normal

$$
\begin{aligned}
& \frac{1}{\sigma r \sqrt{2 \pi}} \exp \left[-(\ln r)^{2} / 2 \sigma^{2}\right] \\
& \sigma>0, r \geq 0
\end{aligned}
$$

Mckay Bessel

$$
\begin{aligned}
& \frac{11-c^{2} 1^{m+1 / 2}}{\pi^{1 / 2} 2^{m} b^{m+1} \Gamma(m+1 / 2)} \exp (-c r / b) r^{m}\left|\begin{array}{l}
\pi I_{m}(r / b) \\
k_{m}(r / b)
\end{array}\right| \\
& b>0, m+1 / 2>0
\end{aligned}
$$$$
\text { range of } r \text { depends on } c
$$ 
Pearson IV

$$
\begin{aligned}
& \frac{1}{\mathrm{aF}(m, v)}\left(1+r^{2} / a^{2}\right)^{-m} \exp \left[-v \tan ^{-1}(r / a)\right] \\
& F(m, v)=\exp (-v \pi / 2) \int_{0}^{\pi} \sin ^{(2 m-2)} \varphi \exp (v \varphi) d \varphi \\
& -\infty<r<\infty
\end{aligned}
$$

We ibull

$$
\begin{aligned}
& \frac{\beta}{\alpha^{\beta}} r^{\beta-1} \exp \left[-(r / \alpha)^{\beta}\right] \\
& r \geq 0 ; \alpha, \beta>0
\end{aligned}
$$

Bivariate Normal Distribution $^{1,7,8}$

This is the distribution that is assumed by most people when they make hand-waving arguments concerning the beam distribution. It is obtained when the particle distributions in the two transverse coordinates are independent and both are normal. The formula given in the table assumes that the radius is measured from the beam centroid so that the means are zero. The parameters are obtained by computing the standard deviations $\sigma_{x}$ and $\sigma_{y}$ in the transverse dimensions, along with the correlation coefficient $\rho$. Figure 5 is a typical fit. to the PARMILA beam. The histogram in this figure, as in others in this paper, was obtained by sorting the radial-particle radius values into 50 bins and plotting a horizontal bar connecting the bin boundaries at the count value. The plot shows that the bivariate normal fails to fit either the peak or the tail of the distribution--falling below each. Chi-square tests confirm that the distribution fits poorly, and comparisons of counted numbers in the tail with the predicted numbers for a complete passage through the linac show that it consistently underestimates the number in the tail by a large amount. 


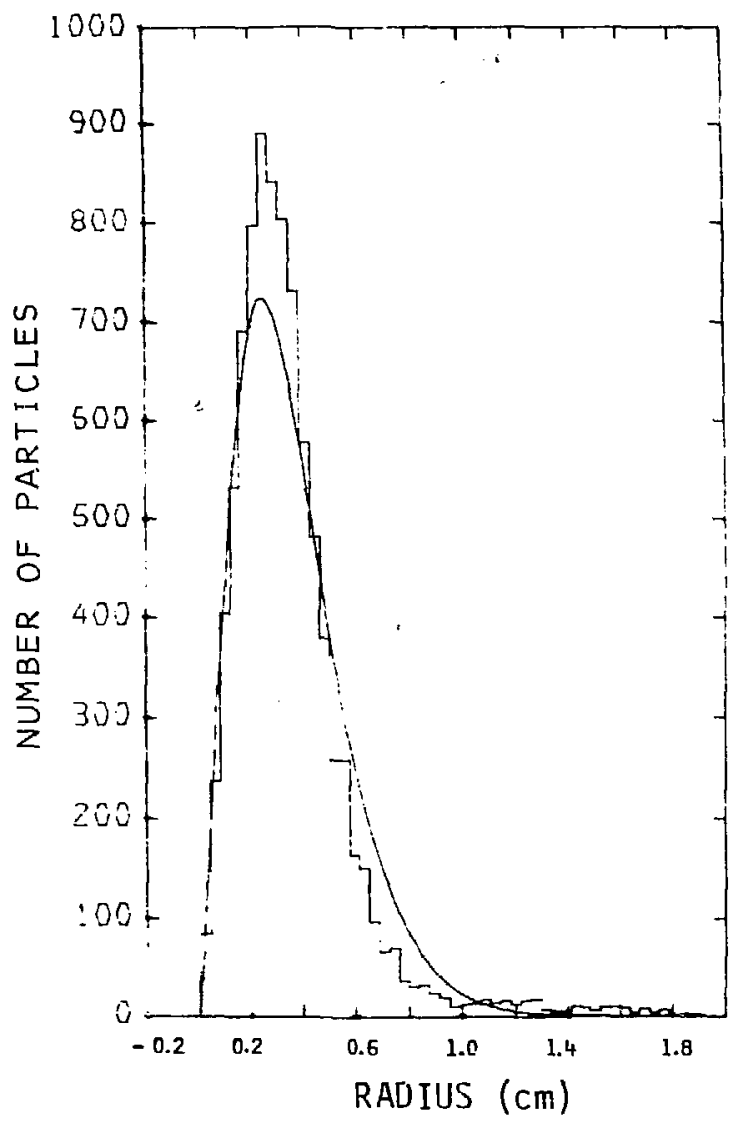

Fig. 5. Typical fit of the bivariate normal distribution to the PARMILA radial particle distribution.
Birnaum-Saunders Distribution 1,9-11

The Birnbaum-Saunders fatiguelife distribution was developed to explain the growth of cracks in mechanical structures under repeated loading. The repeated application of the space-charge impuls? to the particles during the computation somewhat resembles a repeated loading. Maximum likelihood equations were derived for the distribution and were sclved iteratively for the distribution parameters. Maximum likelihood estimations have a number of desirable properties. If efficient estimators exist, then the equations have unique solutions; the solutions, under broad assumptions, converge in probability to the true values of the estimators. Thus, a maximum likelihood estimator is the estimator that minimizes the squared error between itself and the true value. ${ }^{1}$ Generally, the maximuin likelihood equations were used to find distribution parameters for this study.

An example of a fit obtained for the Birnbaum-Saunders distribution, typical of fits obtained at other points along the linac, is shown in Fig. 6 . The height of the peak is overestimated, and the position of the mode (peak) is too close to the axis. The tail is overestimated. When we follow this distribution as it proceeds down the linac, we find that the number in ihe tail almost always is overestimated. The amount of overestimation varies widely from nearly exact to about 40 times too high. Thus, tile Birnbaum-Saunders distribution is not suitable for close caiculations on the tail but could have some use as a bound. 


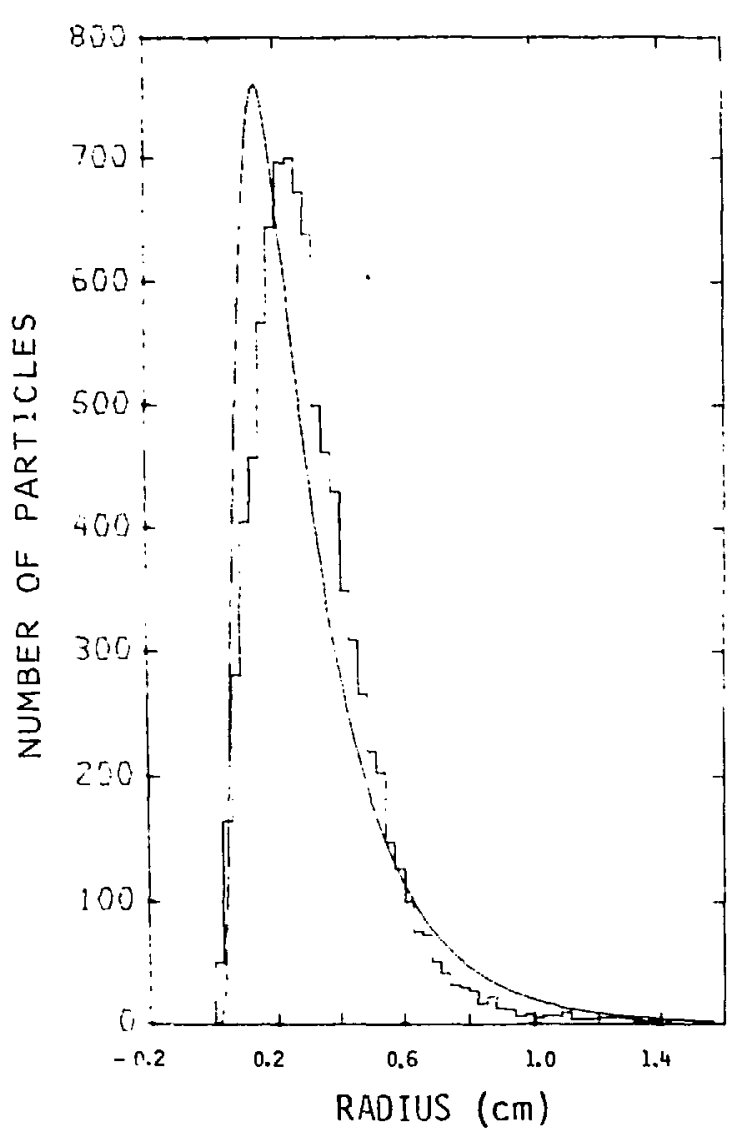

Fig. 6. Tynical fit of the BirnbaumSaunders distribution to the PARMILA radial particle distribution.
Chi Distribution ${ }^{7}$

The chi distribution contains the Rayleigh distribution, whose probability density function (pdf) is given by $\operatorname{pdf}=r \exp \left[-1 / 2(r / \alpha)^{2}\right] / \alpha^{2}$ and the Maxwell distribution, pdf = $4 r^{2} \exp \left(-r^{2} / \alpha^{2}\right) /\left(\alpha^{3} \sqrt{\pi}\right)$, as special cases. The chi-distribution parameters can be estimated either from the moments of the sample or from maximum likelihood equations. The maximum likelihood estimations gave better fits, but the fits, at best, were not as good as either the bivariate normal distribution or the generalized gamma distribution. The chi distribution underestimates both the peak and the tail.

Gamma Distribution ${ }^{1,4,7,8,12}$

The gamma distribution is the Pearson Type III distribution that occupies a line in the skewnesskurtosis plane shown in Fig. 1. The line passes near some of the $\beta_{1}, \beta_{2}$ points assumed by the linac beam.

This distribution contains the exponential and chi-square distributions as special cases. When given as in the table, with its location parameter equai to zero, it also is cailed the Erlang distribution.

Maximum likelihood equations were used to find the distribution parameters. The fits were becter than the bivariate normal fits, but again, they underestimate both the peak and the tail.

General ized Gamma Distribution ${ }^{4,13-16}$

The generalized gamma distribution was introduced by Stacy in $1962 .{ }^{13}$ It covers quite a number of distributions as special cases, including several that 
appear elsewhere in Table I. The distribution seems not to be widely used, probably because no efficient method has been available for the solution of its maximum likelihood equations. During the course of this investigation, an efficient algorithm was developed for the solution of the equations.

The region in the skewness-kurtosis plane covered by the generalized gamma distribution is shown in Fig. 1 . About $80 \%$ of the observed $B_{1}, B_{2}$ points for the PARMILA beam fall inside this region; therefore, good fits are expected. In fact, this distribution gave better fits than any other pure distribution, occasionally providing a satisfactory fit by both chi-squared tests and tail-counting criteria. An example is shown in Fig. 7. Unfortunately, the tail counts predicted by this distribution often tended to be low, especially at higher energies in the linac. The uniformity of the prediction was substantially better than for the Birnbaum-Saunders distribution, but I feit that the predictions were not consistent enough to allow the application of a constant calibrating factor to predict the tail content.

The fact that the generalized gamma distribution can cover a point in the $B_{1}-B_{2}$ plane but still not fit the distribution having those $\beta_{1}-\beta_{2}$ values suggests that the particle distribution may be a mixture of distributions. It does not prove it, because ' 'ne failure to fit may be due to a failure to fit the higher moments. A single, pure distribution might stili be found that hac the proper higher moments.

Generalized Lambda Distribution ${ }^{17}$

This is a four-parameter distribution that covers most of the lower area of the $\mathrm{B}_{1}-\mathrm{B}_{2}$ plane,

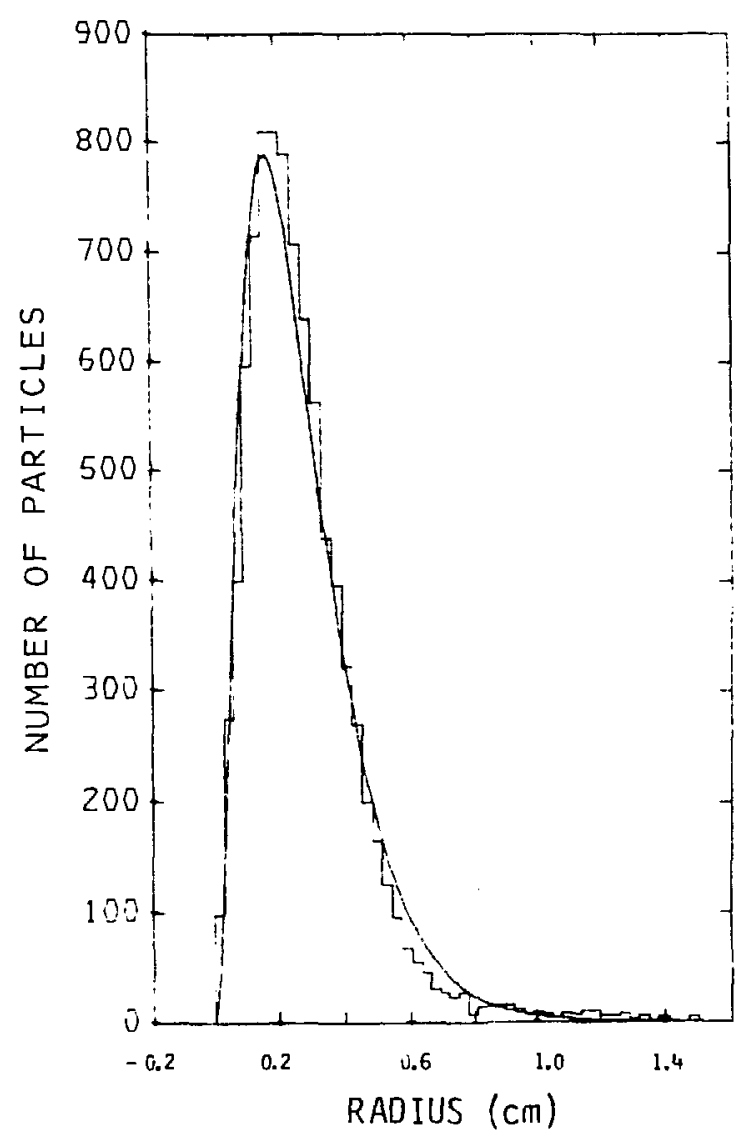

Fiq. 7. Tyoical fit of the pure generalized gamma distribution to the PARMILA radial particle distribution. 
including the region in which the PARMILA radial samples fall. This distribution has two tails; however, one tail can be attenuated enough to approximate the exponential distribution. ${ }^{7}$ Therefore, there should be little problem in getting negligible probability for negative argument values. Examination shows that, for our application, two of the parameters are required to be zero. This condition results in the loss of most of the desirable properties. The fitting and :aluation of this distribution are not straightforward because, given $r$, the second of the equations in the table must be solved for $p$ and then the first equation must he evaluated to $f$ ind $f(p)$. For these reasons, it did not seem very productive to pursue this distribution further. Ramberg et a $1 .{ }^{17}$ mention another asymmetric generalization of the lambda distribution, but I did not collow up this lead.

\section{Gumbel Type I Distribution of the Smallest Extreme $1,2,4$}

This is one of six extreme-value distributions. Only four of them have any possibility of fitting the radial distribution because the other two are zero for large, Dositive values of the argument. The Type I distribution has the range $-\infty<r<\infty$. A fit was made using maximum likelihood equations. It was difficult to obtain convergence in the iterative solution for the distribution Darameters, and the results showed that tine negative tail of the distribution curve was much too large for the distribution to be useful in this application. In fact, the distribution curve seemed to have a more pronounced negative tail than positive.

Gumbe 1 Type I Distribution of the Largest Extreme ${ }^{1,2,4}$

This is the mirror to the previous distribution. It also has the range $-\infty<r<\infty$; however, in this case the negative tail was negligible. The trial fit of the beam distribution was thus substantially better than the Gumbel Type I of smallest extreme, but it grossiy overestimated the height of the peak and the positive tail was almost nonexistent.

Gumbel Type II Distribution of the Largest Extreme ${ }^{1,2,4}$

This distribution has the proper range of $r$. Iterative sclution of the maximum likelihood equations provided the distribution parameters. A fit made at the end of the linac gave a peak of nearly correct height but positioned too close to the axis. The tail was too large but certainly of a bounding nature. 
A second fit at the target; where the beam is approximately an ellipse with aspect ratio $3: 1$, produced a peak too high but a closer bound to the taii. A third fit near the longitudinal center of the linac, however, gave a bound on the tail even looser than the first fit. The bounds on the tail produced by this distribution are large overestimates but they do seem to be less variable than tine estimates produced by the Birnbaum-Saunders distribution. Thus, it might be possible to use this as a bounding distribution and use a calibrating factor to estimate loss.

\section{LaGrange Gamma Distribution $^{18}$}

This is another generalization of the gamma distribution and the distributions are skewed to the right. Differerit distributions are obtained for each value of $j$, the number of terms in the sum. The distributions, once $j$ is fixed, have only two parameters. Two values of $j$ were tried, $j=2$ and $j=3$. The remaining parameters were found using a Nelder-Mead iteration (see Ref. 19) of the maximum likelihood equations. The $j=2$ case underestimated the peak and overestimated the tail. The overestimate did not seem of a bounding nature because the predicted tail was not at a uniform height above the actual tail. The peak position was estimated inside the true position. The $j=3$ case overestimated the peak and badly underestimated the tail. The estimated peak position was at, a smaller radius than the $j=2$ case.

It might be possible to obtain a good fit using higher values of $j$ because the peak and tail values might alternate, but the fact that the peak position moved in the wrong direction makes this unlikeiy. The Nelder-Mead method worked we 11 but slowly. A Newton-method iteration would lead to increasingly complicated equations as $j$ increased, and Newton methods aiways suffer convergence problems if the initial guess is not close enough. This is especially true when, as here, more than one equation must be colved. For these reasons, rio further members of this family were tried.

Log-Normal Distribution 1, 4,7,12

This distribution has only one parameter when we impose the requirement that the probability be zero at the centroid of the beam. Thus, it was a distinct surprise to find that this distribution fit the PARMILA radial distribution at the FMIT target quite well. The fit to the tail appeared to be of a 
bounding nature, uniform, and fairly close. A trial at another position showed that the earlier fit was a fluke because the second fit was very bad; however, the ability to fit at the target may be useful.

Mckay-Bessel Distribution ${ }^{4}=20$

The two McKay-Bessel distributions together cover the entire region below the gamma line in the skewness-kurtosis plane; therefore, these distributions appear to be good candidates. The range of $r$ in these distributions depends on the value of $c$. If $|c|>1$, the modified Bessel function of the first kind, $I_{V}$, is used, and the distribution extends from 0 to $\infty$ if $c>1$ and from $-\infty$ to 0 if $c<1$. When $|c|<1$, the second form using the modified Bessel function of the second $k$ ind, $K_{v}$, is used, and the range is from $-\infty$ to $+\infty$. The case when $|c|>1$ appears to be the one we need, but this distribution covers only a small portion in the $B_{1}-B_{2}$ plane. The maximum likelihood equations for these distributions are very complicated because they involve a derivative of the Bessel function with respect to order. However, Mckay gives equations for the parameters in terms of the moments. The first three moments of the PARMiLA distribution were evaluated and found to lead to a nonreal value of $c$. I concluded that the Bessel distribution moments were not compatible with the radial distribution; hence, no fit was possible.

Pearson Type-IV Distribution ${ }^{4,21}$

The Pearson Type IV distribution covers much of the region in which we found $B_{1}-B_{2}$ points corresponding to the radial distribution (Figs. 1 arid 2 ). This distribution is notorious for its mathematical difficulties. ${ }^{2}$ Bowman and Dusenberry ${ }^{21}$ give the equations for the parameters in terms of the moments. These equations were used to obtain a fit to the radial particle distribution. The Type IV distribution has a range of $-\infty<r<\infty$, and the $f$ it showed a large tail to the left that is incompatible with our requirements. The rest of the fit was better in the right tail than some previously obtained, for example, Gumbel Type I or largest extreme, but was not nearly as good as the generalized gamma distribution.

We ibu11 Distribution ${ }^{1-4,8,12}$

This is, in fact, the Gumbel Type III distribution of the smallest extreme and also is a special case of the generalized gamma distribution. The 
fits obtained were too low in the peak and tail and too high in the region between. The Weibull distribution was tried first because it is known that it is a parent distribution to itself. The results show it is not the right parent.

The list of pure distributions given above is not exhaustive, and there are some candidates that should be checked. The most interesting ones are Bessel distributions of the type discussed by Laha ${ }^{22}$ and used by McNolty et a1.23,24 to describe properties of radar beams. However, the group in the table is fairly representative and covers a good portion of the $B_{1}-\beta_{2}$ plane in which our samples lie. Many in the group are capable of fitting the third and fourth moments of the radial particle distribution. All failed.to fit the radial distribution in one degree or another, the closest one being the generalized gamma distribution that, in one run through the linac, fit the radial distribution in 3 of the 76 cases by a chi-squared criterion. This result suggested that perhaps the disti"ibution I was seeking was not a pure one, and I started to explore the possibility that the radial particle distribution might be reasonably weill fitted by a mixture of distributions.

\section{A DISTRIBUTION THAT WORKS}

The information on the various particle-distribution positions on the Pearson chart combined with the fits obtained using the generalized gamma distribution suggests that something may be wrong with the assumption that only one distribution is involved. Many of the particle distributions lie in the region of the $B_{1}-B_{2}$ plane that the generalized gamma distribution is capable of fitting, yet most of these are not fit well by the generalized gamma using a chi-squared criterion or by comparing predicted-to-counted numbers of particles outside a given radius. This lack of fit suggests that a pure distribution will not work. Examination of a number of particle histograms suggests the presence of a minor peak to the right of the main peak. This secondary peak - Ald have the effect of filling in the region between the main peak and the tail and increasing the number in the tail but still leaving a monomodal distribution. Figure 8 shows an example of how this can happen. Radial distribution histograms with shapes similar to the sum function shown have been observed in the transit of the linac. In fact, Fig. 8 was made using the parameter values obtained from the distribution shown in Fig. 9. 


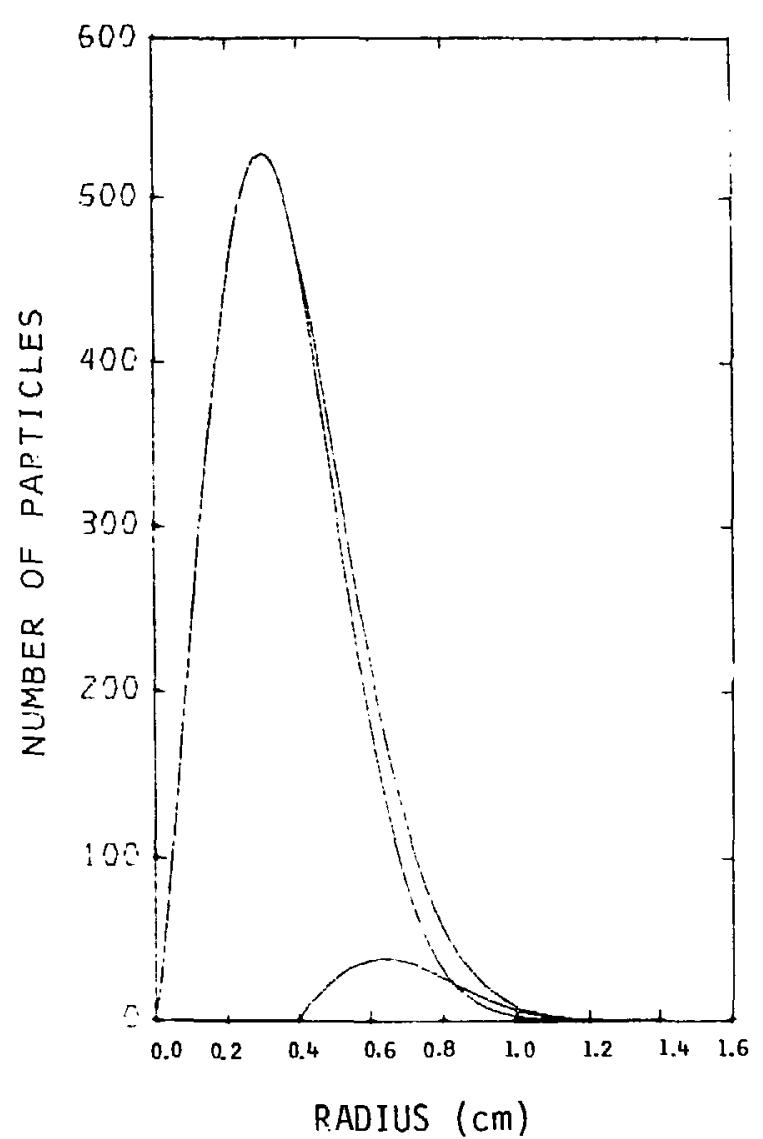

Fig. 8. Example of the result of summing two generalized gamma distributions.

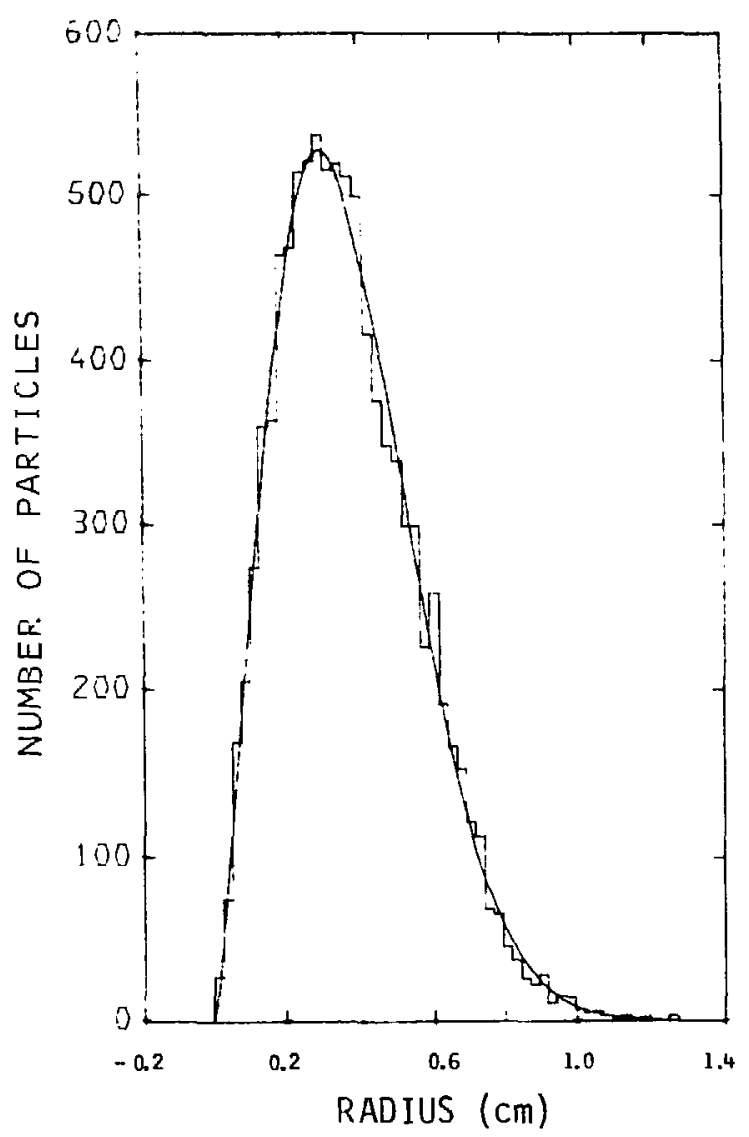

Fig. 9. Example of mixed generalized gamma $f i t$ of a PARMILA radial particle distribution. Fit is good by chi-squared criterion.

It is certainly unlikely that, if more than one distribution is present, there would be a finite number of distributions making up the mixture. More probably, the mixture would consist of a continuous family of distributions with individual members corresponding to different initial radii, that is, different location parameters. We should expect that the use of only two distributions would occasionally result in discontinuities in the fitted curve; in fact, this is the case (see Fig. 10). However, as the example suggests, it might be possible to get a fairly good approximation to the radial particle distribution by using a mixture of only two distributions.

The difficulties accompanying the fitting of mixture distributioris are enormous because each particle must be examined and a decision made as to which 


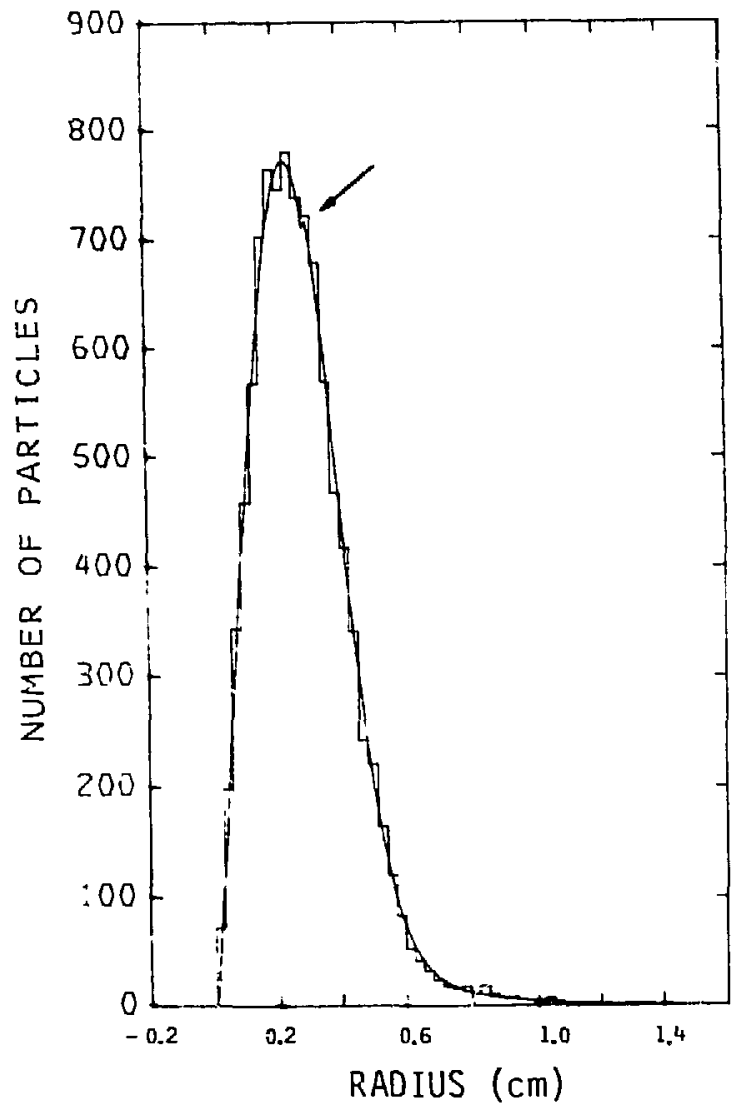

Fig. 10. Example of mixed generalized gamma fit of a PARMILA radial particle distribution. Note discontinuity in the fitting distribution to right of main peak. Fit is good by chi-squared criterion. component distribution it belongs before a fit to the parameters of the component distribution can be made. I decided to pursue this approach anyway and arrived at ar algorithm, to be described below, that accomplishes the fit fairly well ard quite economically. This procedure assumes a mixture of two generalized gamma distributions with one having a positive position parameter relative to the beam centroid.

The fits achieved by this method are quite good. In a particle run generating 76 fits through the FMIT linac, two-thirds of all fits met a chi-squared criterion for goodness of $f i t$, and the remaining ones were, with only two exceptions, only slightly outside the acceptance limit. Two examples of the fits are given in Figs. 9 and 10. Comparison of the histograms with the plots of the fitted function shows that those fits failing to meet the chi-squared criterion usually were due to variation in the peak region of the histogram; Fig. 11 gives an example of such a distribution. No reasonably simple distribution could be expected to fit these variations (not enough parameters); therefore, I feel that the mixed generalized gamma distribution is doing a good job. When the number of particles predicted outside a given radius was compared with the numbers actually present, the results were extremely good. Figure 12 shows how the fitted distribution describes the tail for one run through the FMIT DTL section. It is a plot of the ratio of the number of particles predicted past a given radius $r_{g}$ to the number of particles counted there versus the per cent of the total beam past $r_{g^{*}}$ Five curves are given. The curve marked $A$ is the average over the qualifying cells in the 1 inac. 


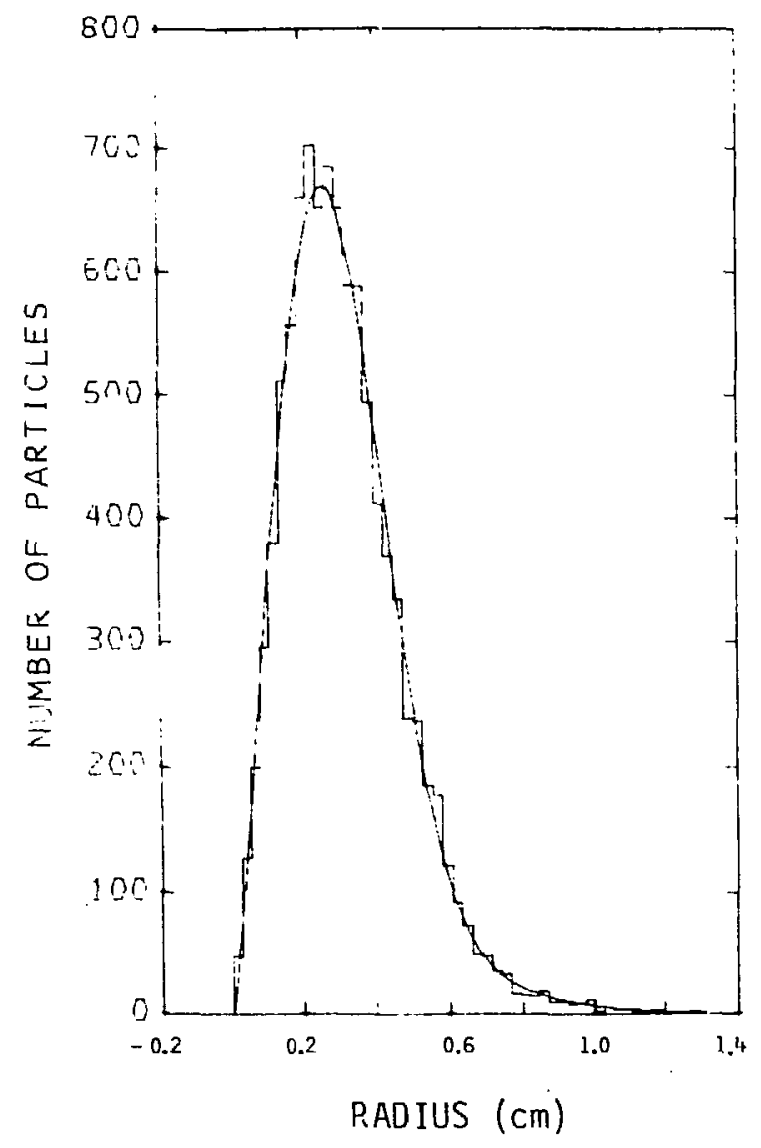

Fig. 11. Example of a mixed generalized gamma fit not good by chi-squared criterion. Failure to meet criterion probably is due to jaggedness of the Deak. The fitted distribution predicted 20 particles past $1 \mathrm{~cm}$; the actual count was 26 .

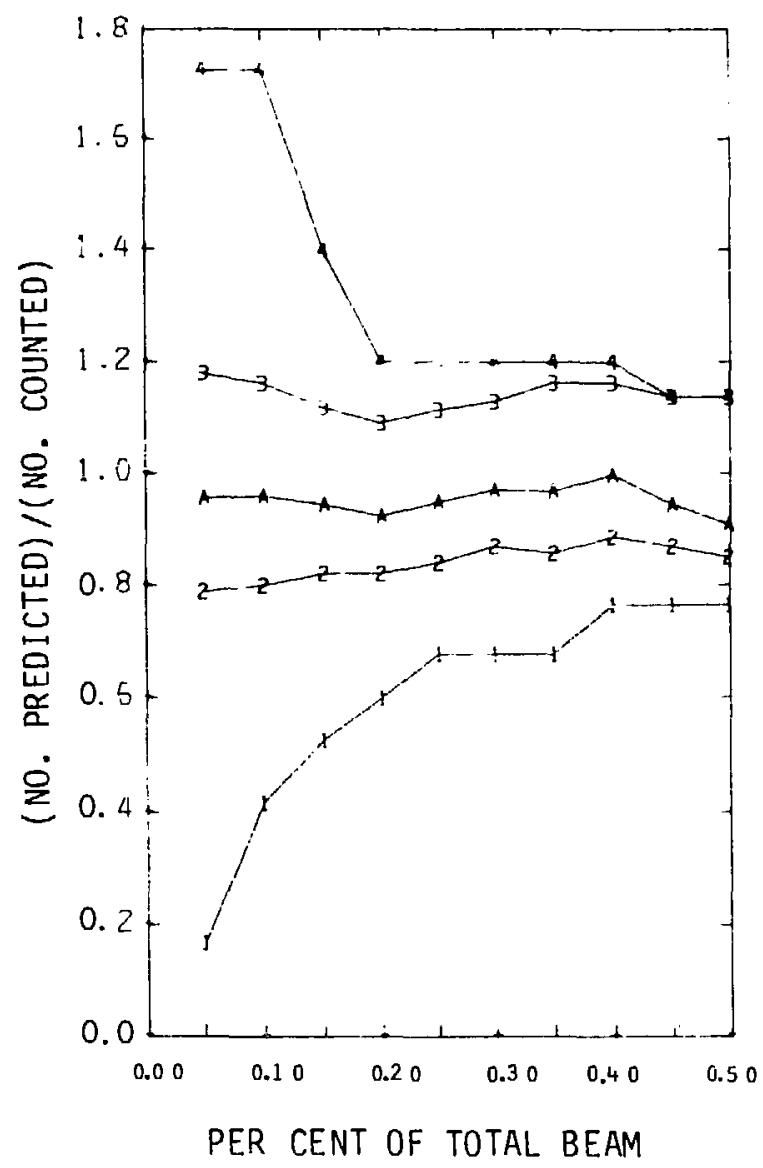

Fig. 12. Ratio of the number predicted to the number counted, plotted against the per cent of the total beam past a fixed radius for one run through the FMIT DTL section. Curve A: average of all qualifying cells; Curve 1: lowest ratio found for qualifying cells; Curve 2: average ratio found for low predictions; Curve 3 : average ratio for high predictions; Curve 4: highest ratio found for qualifying cells.

Curves 2 and 3 qive, respectively, the average underestimate when the prediction is low and the average overestimate when the prediction is high. Curves 1 and 4 are the worst cases seen, that is, the smallest and largest values of the ratio seen for the group of qualifying cells. Figure 12 shows that, on the average, the predicted values are within $\pm 10 \%$ of the exact values and usually are on the low side. The average under- and overestimates are 1ess than $20 \%$. 
As expected. the maximum and minimum of the estimates get larger as the number of particles in the tail decreases. In the example, $0.1 \%$ corresponds to less than 10 particles, and it is hard to believe that one could get very much better absolute accuracy. The relative accuracy should improve when more total particles are used to simulate the beam. The extremes occur mostly in the first cells of the machine when some turbulence is expected that is due to use of the first four quadrupoles for matching. The predictions get better as the beam proceeds tr.rough the linac.

The results given in Fig. 12 suggest that the accuracy of the prediction might be improved by comparing the count of macroparticles to the predicted count and then adjusting the predicted spill by a factor depending on the ratio. This technique might help to cut down on the scatter. At present this has not been tried.

\section{THE MIXED DISTRIBUTION ALGORITHM}

I assume that the mixed distribution is made up of two particle groups, each group having a generalized gamma distribution. Thus, the pdf for the mixed distribution has the form

$$
\begin{aligned}
f(r)= & p\left(\frac{\alpha_{I}}{\beta_{I}}\right)^{\alpha_{I}} \frac{r^{\gamma_{I}} \alpha_{I}-1}{\Gamma\left(\alpha_{I}\right)} \exp \left[-\left(\alpha_{I} / \beta_{I}\right)^{r}\right] \\
& \left.+q\left(\frac{\alpha_{I I}{ }^{\beta_{I I}}}{\beta_{I}}\right)^{\alpha_{I}} \frac{(r-\lambda)^{\gamma_{I I}} \alpha_{I I}{ }^{-1}}{\Gamma\left(\alpha_{I I}\right)} \exp \left[-\left(\alpha_{I I} / \beta_{I I}\right)^{(r-\lambda)}\right)^{\gamma_{I I}}\right] \\
= & p f_{I}(r)+q f_{I I}(r) .
\end{aligned}
$$

The origin of $r$ is assumed to be the beam centroid. Therefore, the location parameter of the second component distribution $\lambda$ is relative to the centroid; $\lambda$ is, of course, assumed positive. I further assume that the lion's share of the particles belongs to the first group, Group I, the group that has a location parameter equal to zero. The assumption that $\lambda$ is positive implies that all particles having a radial position less than $\lambda$ must belong to Group I, and it is this assumption that allows me to separate the two groups and to make the fit. 
Let $N_{I}$ and $N_{I I}$ be the number of particles in Groups I and II, respective$1 y$, and let $N_{\lambda}$ be the actual number of particles inside a radius of $\lambda$. The number of particles in Group I then can be estimated from the relation

$$
N_{I}=\frac{N_{\lambda}}{\int_{0}^{\lambda} f_{I}(r) d r} \text {. }
$$

The function $f_{I}(r)$ is not known, and the mixed distribution algorithm to follow defines an iterative method to find boch $N_{I}$ and $f_{I}(r)$ and to separate the total particles into Groups I and II. Given $N_{I}$, then $N_{I I}, p$, and $q$ are defined by the relations

$$
\begin{aligned}
N_{\text {II }} & =N_{\text {Total }}-N_{I}, \\
p & =N_{I} / N_{\text {Total }}, \text { and } \\
q & =1-p .
\end{aligned}
$$

The second distribution function $f_{I I}(r)$ is obtained by fitting the distribution of particles in Group II.

The algorithm is as follows:

1. Do a fit on the entire set of particles, omitting the $2 \%$ having the largest radii. (Omitting the largest group is not necessary but experience shows that this omission speeds convergence considerably.)

2. Set $\lambda=1.3 \times$ mode; that is, $\lambda=1.3 \times(3 \times$ median $-2 \times \bar{R})$.

3. Define the current $f_{I}(r)$ to be the generalized gamma distribution having the parameters of the previous fit. Ising this $f_{I}(r)$, estimate $N_{I}$ using Eq. (1).

4. Using the current $N_{I}$ and $f_{I}(r)$, separate the particles into Groups $I$ and II as follows:

a. Divide the particle range above $\lambda$ into m sections. All particles with radius at or below $\lambda$ automatically are put in Group I.

b. For each section above $\lambda$, estimate the number of particles that should be in that region, $N_{i}$, using the relation 


$$
N_{i}=N_{I} \int_{r_{i}}^{r_{i+1}} f_{I}(r) d r
$$

c. Count the number of particles actually in the region

$$
N_{i}, r_{i+1}
$$

d. Let $\Delta N_{i}=N_{r_{i}, r_{i+1}}-N_{i}$. If $\Delta N_{i} \geq 1$, remove $\Delta N_{i}$ pariicles from this region and put them in Group II. The particles tc be removed are picked randomly from the range $\left(r_{i}, r_{i+1}\right]$. The remaining particles go into Group $I$.

e. Count the number of particles in Group I, M ${ }_{I}$

5. Fit the new Group I, obtained in Step 4, to determine a new $f_{I}(r)$ and go to Step 3 for the $(j+1)$ th iteration, unless any of the following conditions are fulfilited.

$$
\begin{aligned}
& \text { a. }\left|N_{I}^{(j)}-N_{I}^{(j+1)}\right|<\text { allowed } N_{I} \text { error. } \\
& \text { b. }\left|\left[\gamma_{I}^{(j)}-\gamma_{I}^{(j+1)}\right] / Y_{I}^{(j)}\right|<\text { allowed } \gamma_{I} \text { error. } \\
& \text { c. }\left|M_{I}^{(j+1)}-N_{I}^{(j+1)}\right|<\text { allowed error between count and estimate. }
\end{aligned}
$$

(Because of the random method used to distribute particles between tho two groups, all three criteria are used to prevent excessive iterations or to guard against peculiar samples. The best criterion seems to be $b$, but each of the criteria alone seems to give sufficient accuracy.) If ene of the criteria is fulfilled, exit the iteration and proceed to Step 6 .

6. Fit the current Group II to find $f_{I I}(r)$.

7. Find $p$ and $q$ from Eq. (2).

Note that the choice of $\lambda$ was fairly arbitrary so that there are really only seven fitted parameters, $\alpha_{I}, \beta_{I}, \gamma_{I}, \alpha_{I I}, \beta_{I I}, \gamma_{I I}$, and ${ }_{i}$. The remaining parameters $p, q$, and $N_{I I}$ are determined once $N_{I}$ is found.

The method used to fit the pure generalized gamma distribution is a modified secant method described in the appendix. 
A good value for the allowed $N_{I}$ error seems to be about $0.3 \%$ of $N_{\text {Total }}$. The allowed gamma error can be fairly large, about 0.001 , and still can produce good fits. The error that I have used between predicted $N_{I}$ and counted $M_{I}$ is about $0.1 \%$ of $\mathrm{N}_{\text {Total }}$. The value of $\mathrm{m}$, the number of sections into which the range is divided, was set at 35 after several trials; lower values cause convergence problems because of the randomness of the selection process, and higher values require more computing time per iteration.

VI. USE OF THE MIXED DISTRIBUTION TO PREDICT RELATIVE BEAM LOSS

The amount of current predicted outside the bore radius at a single position in a cell can be considered as a relative measure of the expected beam spill. It is relati:.e in the sense that it should be proportional to the beam spill: thus, it can be compared to a similar prediction made under different operating conditions to decide which condition is more favorable. Because the value is at only one point in the cell and no corrections have been made for regrowth of any tail that might have been wiped off, the measurement can only be relative. However, the predicted current samples the conditions in the cal1, and (for the same linac) the change in this number (accompanying changes in operating parameters such as quadrupole misalignment, field errors, and quadrupole harmonics) should give a quantitative measure of the relative importance of these conditions in producing beam spill.

The estimation of the

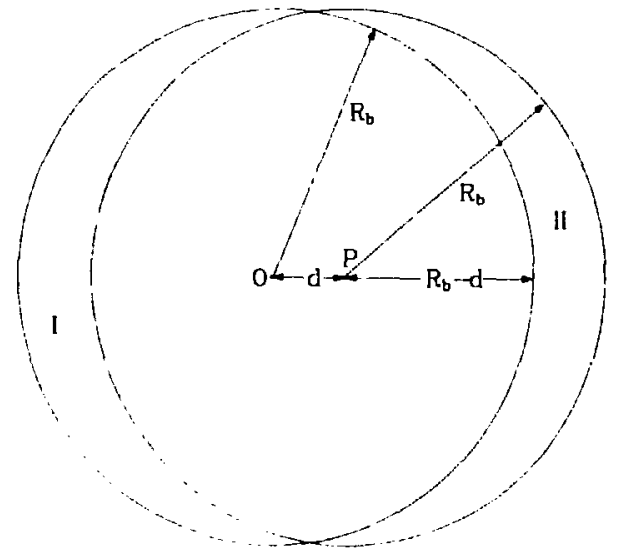

Fiq. 13. Disiributions are caTculated about the centroid of the beam, $P$. The predictions must be corrected to give the loss outside the bore, ahich is centered on 0 . current outside the bore radius, using the radial distribution about the beam centroid, requires a geometric adjustment to find the value outside the bore instead of the value outside the bore radius but centered on the centroid. The simplest way to do this is illustrated in Fig. 13. The losses come from the region outside the circle centered on 0 . The loss 
can be found exactly by calculating the current outside the circle centered on the centroid $P$ then adding the current in Region II and subtracting the currerit in Region I. The required formula for Region II is

$\Delta I_{I I}=\frac{I_{0}}{\pi} \int_{R_{b}-d}^{R_{b}} f(r)\left[\pi-2 \tan ^{-1}\left(\frac{g}{h-R_{b}}\right)\right] d r$,

where

$g(r)=\frac{1}{2}\left(R_{b}+r+d\right)$,

$h(r)=\frac{\left(g-R_{b}\right)(g-r)(g-d)}{g}$, and

$d$ is the radial distance from the axis of the machine to the beam centroid. The formula for Region $I$ is similar, but it is probably all right to neglect Region I because its average distance from the centroid is greater; thus, the amount of current in Region I is smaller and its neglect gives an overestimate of the relative spill.

- Figure 14 shows the amount of current predicted outside the bore as a function of cell for a run through the FIMIT linac under ideal conditions.

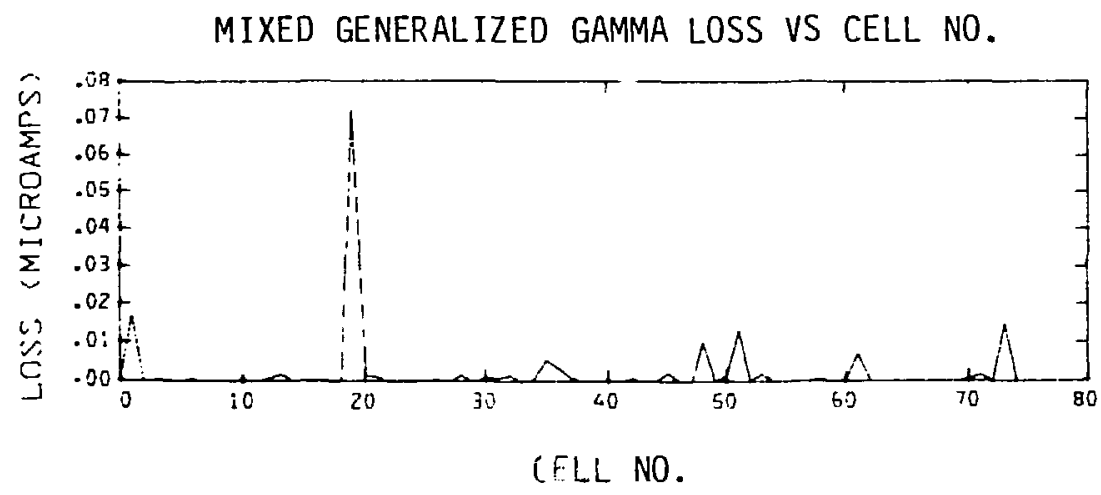

Fig. 14. Loss prediction for a perfect FMIT OTL section. Input beam is from F-17 RFQ computed with time as the independent variable. 
Figure 15 is a similar plot for the same linac when some quadrupole misalignments and field errors are included in the calculation. The increase in loss is evident. Figure 16 shows the loss from a perfectly aligned FMIT linac that is due to the inclusion of $2 \% \mathrm{~N}=6$ harmonics in all the quadrupoles. There is little change from Fig. 14. This result is in agreement with Gluckstern's prediction for periodic systems. ${ }^{25}$

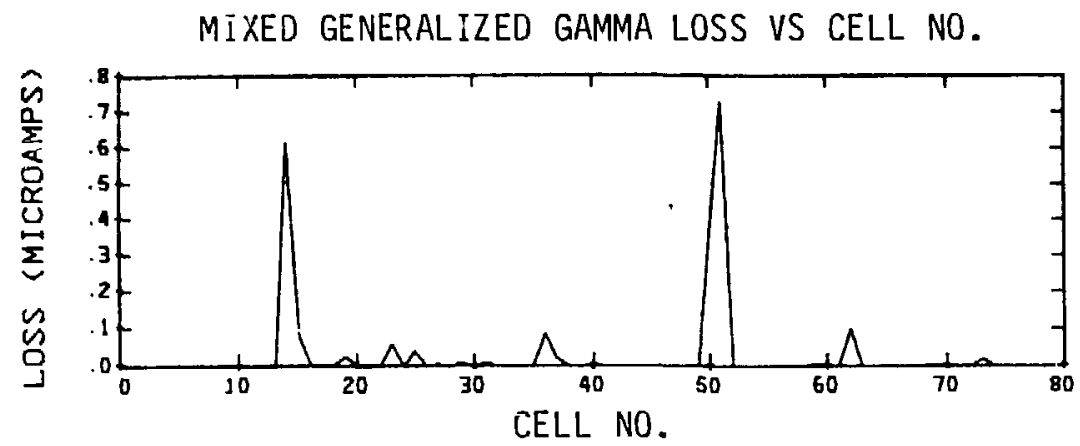

Fig. 15. Loss prediction for the FMIT DTL section having random misalignments and field errors. Input is the same as in Fig. 14. Note that the vertical scale is 10 times the scale of Fig. 14.

MIXED GENERAL IZED GAMMA LOSS VS CELL NO.

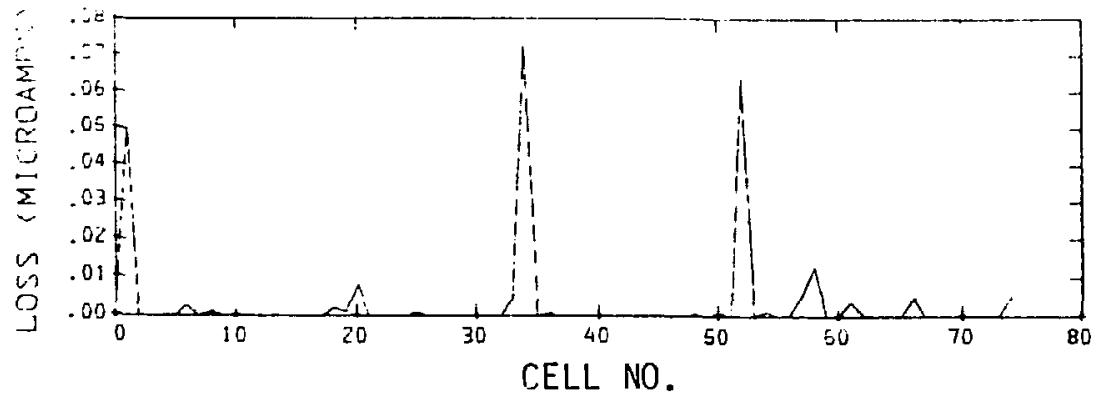

Fig. 16. Loss prediction for FMIT DTL section with $2 \% \mathrm{~N}=6$ quadrupole harmonics. Compare with Fig. 14 . 
The change in current between Figs. 17 and 18 describes the effect of using two different RFQ designs to feed the FMIT DTL section. This DTL section uses the first four quadrupoles to provide a transverse match between the input beam and the section. The plots in the figures were obtained by determining the proper quadrupole strengths to match the RFQ useu, setting the quadrupoles in the DTL section to these values, and then passing the beam through the section. The beam from the round-vane RFQ clearly gives a better result.

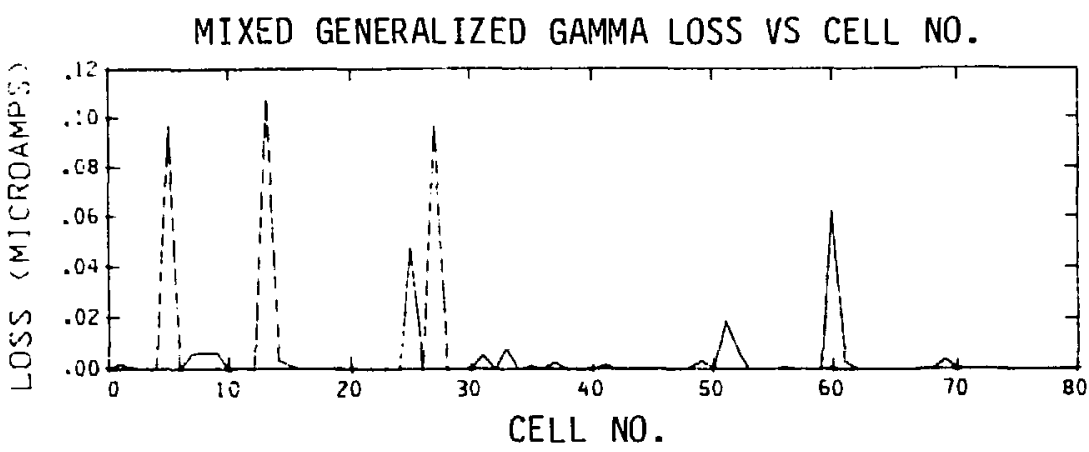

Fig. 17. Loss prediction for a perfect FMIT DTL section; input beam is from F-17 RFQ computed with $Z$ as the independent variable.

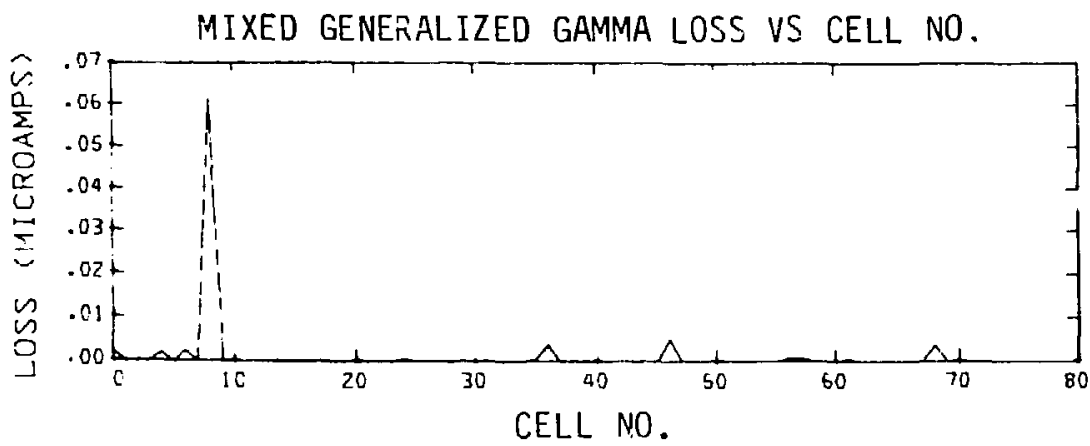

Fig. 18. Loss prediction for a perfect FMIT DTL section; input beam is from round-vane RFQ computed with $Z$ as the independent variable. 


\section{QUANTITATIVE PREOICTION OF BEAM SPILL}

In a real beam inside a bore tube, losses occur because particles, in some manner, migrate to the tube wall and strike it. It is fairly $\mathrm{s}$.fe to assume that in the gap the losses are negiigible, but that the migration past the bore radius continues as the bunch crosses the gap. As the bunch starts into the next bore tube, the particles outside the bore radius $R_{b}$ start to be wiped off until all the particles that migrated past $R_{b}$ are removed. It seems a reasonable assumption that the number of particles migrating past $R_{b}$ in unit time is constant, provided that the particle distribution is constant. If we sample the distribution only once per cell, we have no choice but to assume either that it is constant or that it varies linearly across the cell. In either event, if we know the distributions, the calculation of the losses deDends only on the migration rate of particles past the bore radius. This rate becomes a calibrating factor for the particle simulation code. The measurement of this factor in a real machine would provide the calibrations and would be a test of the validity of this type of argument.

In the examples given in Sec. VI, the losses correspond to the assumption that the distribution is constant across the cell and that the migration is at a rate exactly correct to re-establish the tail in one rf period.

In the simulation, no macroparticle losses can occur, because (if they did) our tail would be clipped, the fitted distributions would reflect this, and the predictions would be too low. Thus, this technique is not useful in examining losses that are due to particles outside the bucket and anderlying assumption of the method is that the losses in the machine are small compared to the total current. The losses that occur because of particles outside the bucket can be handled quite well in standard PARMILA runs merely by observing the number and location of macroparticle losses.

\section{SUMMARY AND CONCLUSIONS}

At present, there is no numerical method for estimating beam spill in linacs. One way to accomplish an estimate depends on fitting the radial particle distribution produced by a simulation code to a probability distribution. Manipulation of the fitting distribution will allow estimates of the amount of beam lost.

I have examined the properties of the PARMILA code's radial distribution as they relate to the probability distribution properties required to fit it 
and have tried a fairly broad class of probability distributions. The generalized gamma distribution has proved to be the best of the pure distributicns in fitting the radial distribution, blii it does not provide a good enough fit.

I explored the possibility that a mixture of two such generalized gamma distributions might give a satisfactory fit and developed an algorithm to separate the two component distributions and to fit the PARMILA radial distribution. The fits obtained are not always as good as might be desired but seem to predict the radial distribution tail to within, at worst, a factor of 2 or 3. The fits usually are much becter than this; therefore, I feel that the mixed distribution can be used for predictive purposes.

I have given examples of the use of the fitted distribution to predict the relative effects of misalignment, quadrupole harmonics, and injection conditions.

The problems in using the fitted distribution to predict quantitative beam losses were explored briefly.

The statistical method developed here is very powerful in handling lack of numerical resolution, one of the two major problems in estimating beam spill with any physical model. The other problem is whether or not the physical model itself is adequate.

\section{ACKNOWLEDGMENTS}

The author would like to thank R. A. Jameson and R. K. Cooper for helpful comments and encouragement. 


\section{APPENDIX}

FITTIN'G THE GENERAL IZED GAMMA DISTRIBUTION

The maximum likelihood equations for the generalized gamma distribution are

$\ln \alpha-\ln \beta-\omega(\alpha)+\frac{\gamma}{n} \sum_{i}^{n} \ln x_{i}=0$,

$B-\frac{1}{n} \sum_{1}^{n} x_{i}^{\gamma}=0 \quad$, and

$B+\frac{\alpha \beta \gamma}{n} \sum_{i}^{n} \ln x_{i}-\frac{\alpha \gamma}{n} \sum_{i}^{n} x_{i}^{\gamma} \ln x_{i}=0$.

In Eq. $(A-1), \psi(\alpha)$ is the psi or digamma function--the logarithmic derivative of the gamma function. ${ }^{26}$

Equation (A-2) gives $B$ directly in terms of $\gamma$ and allows the elimination of $B$ from Eqs. $(A-1)$ and $(A-3)$. After this substitution, Eq. $(A-3)$ proves to be soluble for $\alpha$ in terms of $\gamma$. The following set then becomes the system to solve.

$x=\frac{n \sum_{1}^{n} x_{i}^{\gamma}}{\gamma\left(n \sum_{1}^{n} x_{i}^{\gamma} \text { on } x_{i}-\sum_{1}^{n} x_{i}^{\gamma} \cdot \sum_{i} \text { on } x_{i}\right)}$,

$B=\frac{1}{n} \sum_{1}^{n} x_{i}^{\gamma}, \quad$ and

$n \equiv 2 \ln n-\ln \gamma-\psi[\alpha(\gamma)]-\ln \left(n \sum_{1}^{n} x_{i}^{\gamma} \ln x_{i}-\sum_{1}^{n} x_{i}^{\gamma} \cdot \sum_{1}^{n} \ln x_{i}\right)$

$$
+\frac{\gamma}{n} \sum_{1}^{n} 2 n x_{i}=0
$$


To solve this set, it is necessary only to iterate on $\gamma$ because Eq. (A-4) gives the required value of $\alpha(\gamma)$ for the digamma function. Once a value of $\gamma$ is found that satisfies Eq. $(A-6)$, Eqs. $(A-4)$ and $(A-5)$ give the other two parameters.

The sqlution of Eq. (A-6) is obtained by using a secant method with a convergence factor, $c$. Iteration is performed with the equation

$\gamma_{i+1}=\gamma_{i}-c \frac{\left(\gamma_{i}-\gamma_{i-1}\right)}{\left(h_{i}-h_{i-1}\right)} h_{i}=\gamma_{i}-c\left[\left(\gamma_{i}-\gamma_{i-1}\right)\left(\frac{h_{i}}{h_{i-1}}\right)\right] /\left(1-\frac{h_{i}}{h_{i-i}}\right) \quad$ (A-7)

The second form can be used to prevent division by 0 if $h_{i}$ is too close to $h_{i-l}$. The equation requires initial guesses for $\gamma_{1}$ and $\gamma_{0}$. In the computations on the FMIT linac, $\gamma_{0}=3.0$ and $\gamma_{1}=0.99 \times \gamma_{0}$ work well for the first call. If the fitting routine is called in an iterative way, as described in the body of the paper, then the final value from the previous fit is user for $\gamma_{0}$. The quantity $h_{0}$, of course, can be evaluated from Eq. $(A-6)$ but setting it equal to

$h_{0}=2 \ln n+\frac{\gamma_{1}}{n} i_{1}^{n}$ in $x_{i}-\ln \gamma_{1}$

before entry into the iteration loop is simpler and gives satisfactory results; $h_{1}$ is, of course, always calculated in the loop.

If the initial guess for $\gamma_{1}$ is too far below the true value of $\gamma$, the iteration will approach the value $\gamma_{\infty}=0$. This situation is prevented by checking to see if $\mathrm{r}_{j+l}$ falls below a prescribed value and restarting the iteration at a higher value of $\gamma_{1}$ when it occurs. In such cases, a reduced value of $c$ also is used.

Good values for $c$ lie between 0.6 and 0.8 unless $\gamma_{1}$ is known to be close to the true value; in which case, convergence is speeded by a choice of $c=1$. 


\section{REFERENCES}

I. N. R. Mann, R. E. Schafer, N. D. Singpurwalla, Methods for Statistical Analys is of Reliability and Life Data (John Wiley \& Sons; New York, 1974).

2. E. J. Gumbe1, Statistics of Extremes (Columbia University Press, New York, 1958).

3. G. P. Boicourt and R. A. Jameson, Pror. 1979 Linear Accelerator Conf., Montauk, New York, September 10-14, 1979, Brookhaven National Laboratory report BNL-51134, p. ?38 (1980).

4. N. L. Johnson and S. Kotz, Continuous Univariate Distributions - 1 (John Wiley \& Sons, New York, 1970$)$.

5. M. R. Spiegel, Theory and Problems of Statistics (Schaum's Outline Series, McGraw Hill Book Company, New York, 1961).

6. G. P. Boicourt, R. A. Jameson, and R. S. Mills, Proc. 1981 Linear Accelerator Conf., Santa Fe, iN, Los Alamos National Laboratory report LA-9234-C, p. 36 (February 1982).

7. E. Parzen, Modern Probability Theory and Its Applications (John Will" \& Sons, New York, To60).

8. W. Feller. An Introduction to Probability Theory and Its Applications (.John Wiley Sons, New Yarki 1971) Vol, II.

9. 2. W. Birnbaum and S. C. Saunders, SIAM J. Appl. Math., Vol. 16, No. 3, pp. 637-652. (May 1968).

10. Z. W. Birnbaum and S. C. Saunders, J. Appl. Prob. 6, pp. 319-327 (1969).

11. Z. W. Birnbaum and S. C. Saunders, J. App 1. Prob. 6, pp. 328-347 (1969).

12. R. E. Barlow and F. Proschan, Mathematical Theory of Reliability (John Wiley \& Sons, New York, 1965).

13. E. W. Stacy, Ann. Math. Statistics 33, pp. 1187-1192 (1962).

14. E. W. Stacy and G. A. Mihram, Technometrics, Vol. 7, No. 3, pp. 349-358 (1955).

15. H. Leon Harter, Aerospace Research Laboratories report ARL66-0158, WrightPatterson AFB, Ohio, (August 1966).

16. H. Leon Harter, Technometrics, Vol. 9, No. 1, pp. 159-165 (February 1967).

17. J. S. Ramberg, E. J. Dudewicz, P. R. Tadikamalla, and E. F. Mykytka, Technometrics, Vol. 21, No. 2 (May 1979). 
18. D. L. Nelson and P. C. Consul, "A LaGrangian Gamma Distribution," Statistical Distributions in Scientific Work (D. Reidel Publishing Co., Boston, T975) Vol. T, pp. 24T-246.

19. D. M. Himmelblau, Applied Nonlinear Programming (McGraw-Hill Book Company, New York, 1972).

20. A. T. McKay, Biometrica 24, pp. 39-44 (1932).

21. K. 0. Bowman and W. E. Dusenberry, "Transformation of the Pearson System with Special Reference to Type Iv," Statistical Distributions in Scientific Work (D. Reidel Publishing Company, Boston, 1975) VoT. 1, pp. 381390 .

22. R. G. Laha, Calcutta Mathematical Society Bulletin, Vol. 46, pp. 59-72 (1953).

23. F. McNolty, Sankhya: The Indian Journal of Statistics: Series B, 29, pp. $235-248(1967)$.

24. F. McNolty, J. R. Huynen, and E. Hansen, "Certain Statistical Distributions Involving Special Functions and Their Applications, "Statistical Oistributions in Scientific Hork (D. Reidel Publishing Company, Boston, 1975) VoT. T, pp. 131-160.

25. R. L. Gluckstern, Los Alamos National Laboratory report LA-8526-MS (September 1980).

26. M. Abramowitz and I. A. Stegun, Handbook of Mathematical Functions with Formulas, Graphs, and Mathematical Tables (Dover Publications, 1956) Chap. 6, p. 258 . 